\title{
TAIL PROBABILITY OF THE SEQUENTIAL MAXIMUM LIKELIHOOD ESTIMATOR FOR THE EXPONENTIAL CLASS
}

\author{
Haruyoshi Mita*
}

\begin{abstract}
In this paper we will study the asymptotic behavior of tail probability of the sequential maximum likelihood estimator for the exponential class. This class contains many important stochastic processes including the Wiener process, Bernoulli process, Poisson process, gamma process, and Gaussian autoregressive process, etc. We introduce a stopping time which is useful in sequential statistical analysis. It is shown that the tail probability of the sequential maximum likelihood estimator based on the stopping time decreases exponentially fast as the stopping boundary diverges, and some examples are verified.
\end{abstract}

\section{Introduction and preliminaries}

Let $Z(t), t \in T$, denote a stochastic process defined on the probability space $\left(\Omega, F, P_{\theta}\right)$, where $T=[0, \infty)$ or $\{1,2, \cdots\}$. The probability $P_{\theta}$ depends on a parameter $\theta$, and $\theta$ takes its values in an open set $\Theta \subset R^{1}$. Let $\boldsymbol{F}_{t}, t \in T$, be the $\sigma$-field generated by the process $Z(s), s \leq t$. The restriction of $P_{\theta}$ to the $\sigma$-field $\boldsymbol{F}_{t}$ is denoted by $P_{\theta, t}$. We assume that the probability $P_{\theta, t}, \theta \in \Theta$, $t \in T$, is dominated by the restriction of a $\sigma$-finite measure $\mu$ to $\boldsymbol{F}_{t}$, which is denoted by $\mu_{t}$, and the likelihood function as Radon-Nikodym derivative is given in the form

$$
L_{t}(\theta)=\frac{d P_{\theta, t}}{d \mu_{t}}=g_{t} \exp (\theta X(t)-f(\theta) Y(t))
$$

where $g_{t}, X(t)$ and $Y(t)$ are $\boldsymbol{F}_{t}$-measurable real valued stochastic processes and $P_{\theta}\left(g_{t} \geq 0\right)=P_{\theta}(Y(t)>0)=1$ for all $\theta \in \Theta$. Moreover, we assume that $g_{t}, X(t)$, and $Y(t)$ have right continuous paths and left limits in continuous time parameter case and $f(\theta)$ is a twice differentiable real valued function and $\ddot{f}(\theta)>0$ for all $\theta \in \Theta$. When a stochastic process $Z(t)=(\mathrm{X}(t), \mathrm{Y}(t)), t \in T$, fulfills the above conditions, we shall say that the stochastic process $Z(t), t \in T$, belongs to the exponential class.

Let $\tau$ be an arbitrary stopping time, i.e., $\tau$ is a random variable defined on $\Omega$ and takes its values in $T \cup\{\infty\}$, and $\{\omega \in \Omega, \tau(\omega) \leq t\} \in F_{t}$. Then the $\sigma$ field of the $\tau$-past of the process $Z(t)$ is defined by

$$
\boldsymbol{F}_{\mathrm{r}}=\left\{A \in \boldsymbol{F}: A \cap\{\omega: \tau(\omega) \leq t\} \in \boldsymbol{F}_{\boldsymbol{t}} \text { for all } t \in T\right\} .
$$

In this paper we shall consider only finite stopping times, i.e., $P_{\theta}(\tau<\infty)=1$ for each $\theta \in \Theta$.

Let $P_{\theta, \tau}$ and $\mu_{\mathrm{r}}$ denote the restrictions of $P_{\theta}$ and $\mu$ on $\boldsymbol{F}_{\tau}$, respectively. Then, by Sudakov's lemma, $P_{\theta, r}$ is dominated by $\mu_{\tau}$, and the likelihood func-

Received May, 1995. Revised July, 1995. Accepted August, 1995.

* Faculty of Liberal Arts, University of The Sacred Heart, Shibuya-ku, Tokyo 150, Japan 
tion is given by

$$
L_{\tau}(\theta)=\frac{d P_{\theta, \tau}}{d \mu_{\tau}}=g_{\tau} \exp (\theta X(\tau)-f(\theta) Y(\tau))
$$

(c.f., Basawa and Prakasa Rao (1980), and Różański (1980)). Let $\hat{\theta}_{\tau}$ be the maximum likelihood estimator for the stopped likelihood function $L_{\mathrm{r}}(\theta)$. We have $\dot{f}\left(\hat{\theta}_{\tau}\right)=X(\tau) / Y(\tau)$.

Now we introduce the following stopping time

$$
\tau_{a, b}(u)=\inf \{t: a X(t)+b Y(t) \geq u\},
$$

where $a \neq 0, b$, and $u>0$ are constants, and $a$ and $b$ are chosen such that $P_{\theta}\left(\tau_{a, b}<\infty\right)=1$ for all $\theta \in \Theta$. The stopping time defined by (1.3) is often used for sequential statistical inference of the exponential class (1.1). For instance, it appears in sequential estimation problems for the exponential class of processes with independent increments (see, Winkler and Franz (1979), Winkler, Franz, and Küchler (1982), and Stefanov (1985)). Furthermore, the stopping time for a one sided sequential probability ratio test is defined by the form (1.3) (c.f., Wald (1947)). We often write $\tau(u)=\tau_{a, b}(u)$ or $\tau=\tau_{a, b}(u)$ for convenience. We denote $D_{\tau}(u)$ as the overshoot for the stopping time (1.3), i.e.,

$$
D_{\tau(u)}=a X(\tau)+b Y(\tau)-u .
$$

In general, we denote $\varphi_{\boldsymbol{X}, \theta}(s)$ as the moment generating function (m.g.f.) of a random variable $X$ under $P_{\theta}$.

Sørensen (1986) showed the consistency and the asymptotic normality of $\hat{\theta}_{\mathrm{r}(u)}$ under the conditions such that $D_{\tau(u)}$ is ignored for large $u$. We state here his results without proof (for details, see Sørensen (1986)).

Theorem 1.1. (Sørensen) Suppose that $P_{\theta}(\tau(u)<\infty)=1$ for all $\theta \in \Theta$.

(i) (consistency) If there exists a neighbourhood $S$ of 0 such that for any fixed $s_{0} \in S, \varphi_{D_{r}, \theta}\left(s u^{-1}\right) \rightarrow 1$ as $u \rightarrow \infty$ uniformly for $\left(\theta^{\prime}, s\right)$ in a neighbourhood of $\left(\theta, s_{0}\right)$. Then we have

$$
\hat{\theta}_{r(u)} \rightarrow \theta \text { in probability as } u \rightarrow \infty \text { under } P_{\theta} .
$$

(ii) (asymptotic normality) If there exists a neighbourhood $S$ of 0 such that for any fixed $s_{0} \in S, \varphi_{D_{r}, \theta^{\prime}}\left(s u^{-1 / 2}\right) \rightarrow 1$ as $u \rightarrow \infty$ uniformly for $\left(\theta^{\prime}, s\right)$ in a neighbourhood of $\left(\theta, s_{0}\right)$. Then we have

$$
\sqrt{\frac{u \ddot{f}(\theta)}{a \dot{f}(\theta)+b}}\left(\hat{\theta}_{\tau(u)}-\theta\right) \rightarrow N(0,1) \text { in law as } u \rightarrow \infty \text { under } P_{\theta} \text {. }
$$

We assume the following condition:

(C) For any $\theta \in \Theta$, there exists a neighbourhood $N_{\theta}$ of $\theta$ and a random variable $M_{u, \theta}$ such that for all $u>0$ and all $\theta^{\prime} \in N_{\theta}$,

$$
P_{\theta^{\prime}}\left(D_{\tau(u)} \leq M_{u, \theta)}=1\right. \text {, }
$$

where the distribution of $M_{u, \theta}$ under $P_{\theta^{\prime}}$ is independent of $u$ and $\theta^{\prime} \in N_{\theta}$, and 
the moment generating function of $M_{u, \theta}$ under $P_{\theta}$ exists in a neighbourhood of origin.

If condition (C) is satisfied then there exists a positive number $s_{1}$ such that for any $s \in\left[-s_{1}, s_{1}\right]$ and any $\delta>0$,

$$
-s_{1} u^{-\delta} M_{u, \theta} \leq s u^{-\delta} D_{\tau} \leq s_{1} u^{-\delta} M_{u, \theta} .
$$

Hence it follows that

$$
\int \exp \left(-s_{1} u^{-\delta} M_{1, \theta}\right) d P_{\theta} \leq \varphi_{D_{r}, \theta^{\prime}}\left(s u^{-\delta}\right) \leq \int \exp \left(s_{1} u^{-\delta} M_{1, \theta}\right) d P_{\theta}
$$

for all $\theta^{\prime} \in N_{\theta}$ and all $u>1$.

Using dominated convergence theorem we obtain

$$
\varphi_{D_{\mathbf{r}}, \theta^{\prime}}\left(s u^{-\delta}\right) \rightarrow 1 \text { as } u \rightarrow \infty
$$

uniformly for $\left(\theta^{\prime}, s\right) \in N_{\theta} \times\left[-s_{1}, s_{1}\right]$.

Therefore, if condition (C) is satisfied then the conditions in (i) and (ii) of Theorem 1.1 are satisfied. This leads us to the next corollary.

Corollary 1.1. Suppose that $P_{\theta}(\tau(u)<\infty)=1$ for all $\theta \in \Theta$. If condition $(C)$ is fulfilled then for any $\theta \in \Theta$, we have

$$
\hat{\theta}_{\tau} \rightarrow \theta \text { in probability as } u \rightarrow \infty \text { under } P_{\theta} .
$$

\section{Main result}

We put $l_{\mathrm{r}}(\theta)=\log L_{\mathrm{r}}(\theta)$ and $h(\theta)=f(\theta)+a^{-1} b \theta . \quad$ By virtue of (1.2) and (1.4), we have for any $\theta \in \Theta$,

and

$$
a E_{\theta}(X(\tau))+b E_{\theta}(Y(\tau))=E_{\theta}\left(D_{\mathrm{r}}+u\right)
$$

By (2.1) and (2.2), we have $(a \dot{f}(\theta)+b) E_{\theta}(Y(\tau))=E_{\theta}\left(D_{\mathrm{r}}+u\right)$. We have $a \dot{h}(\theta)=$ $a \dot{f}(\theta)+b>0$, because $P_{\theta}(Y(t)>0)=1$ for all $\theta \in \Theta$. Therefore, $h$ is invertible on $\Theta$.

Let $K_{u}\left(\theta^{\prime}, \theta\right)$ be the Kullback-Leibler information distance from $P_{\theta^{\prime}, \tau}$ to $P_{\theta, r}$, i.e.,

$$
K_{u}\left(\theta^{\prime}, \theta\right)=\int \log \frac{d P_{\theta^{\prime}, \mathrm{r}}}{d P_{\theta, \tau}} d P_{\theta^{\prime}, \tau} .
$$

By (2.1) and (2.2), we have

$$
\begin{aligned}
K_{u}\left(\theta^{\prime}, \theta\right) & =\int\left(\left(\theta^{\prime}-\theta\right) X(\tau)-\left(f\left(\theta^{\prime}\right)-f(\theta)\right) Y(\tau)\right) d P_{\theta^{\prime}, r} \\
& =\left(\left(\theta^{\prime}-\theta\right) \frac{\dot{f}\left(\theta^{\prime}\right)}{a \dot{f}\left(\theta^{\prime}\right)+b}-\left(f\left(\theta^{\prime}\right)-f(\theta)\right) \frac{1}{a \dot{f}\left(\theta^{\prime}\right)+b}\right) E_{\theta^{\prime}}\left(D_{r}+u\right) .
\end{aligned}
$$

By condition $(C)$, we have

$$
\lim _{u \rightarrow \infty} \frac{1}{u} K_{u}\left(\theta^{\prime}, \theta\right)=\frac{\left(\theta^{\prime}-\theta\right) \dot{f}\left(\theta^{\prime}\right)-\left(f\left(\theta^{\prime}\right)-f(\theta)\right)}{a \dot{h}\left(\theta^{\prime}\right)} .
$$


We put

$$
K\left(\theta^{\prime}, \theta\right)=\frac{\left(\theta^{\prime}-\theta\right) \dot{f}\left(\theta^{\prime}\right)-\left(f\left(\theta^{\prime}\right)-f(\theta)\right)}{a \dot{h}\left(\theta^{\prime}\right)} .
$$

Theorem 2.1. Suppose that $P_{\theta}\left(\tau_{a, b}(u)<\infty\right)=1$ for all $\theta \in \Theta$ and condition (C) is fulfilled. Then for any $\theta \in \Theta$ and $\epsilon>0$ such that $\theta \pm \epsilon \in \Theta$ it follows that

$$
\lim _{u \rightarrow \infty} \frac{1}{u} \log P_{\theta, \tau}\left(\left|\hat{\theta}_{\tau}-\theta\right|>\epsilon\right)=-B(\theta, \epsilon),
$$

where $B(\theta, \epsilon)=\min \{K(\theta+\epsilon, \theta), K(\theta-\epsilon, \theta)\}$.

We will postpone the proof of this theorem to the next section.

\section{Lemmas}

We need some lemmas to prove Theorem 2.1 .

Lemma 3.1. Suppose that $P_{\theta}\left(\tau_{a, b}(u)<\infty\right)=1$ for all $\theta \in \Theta$ and condition (C) is fulfilled. Then for all $\epsilon>0$ and s such that $\operatorname{sh}(\theta+\epsilon)+h(\theta) \epsilon h(\Theta)$, we have

$$
\varphi i_{r}(\theta+\varepsilon), \theta(s)=\exp \left(a^{-1} u\left(s+\theta-\theta^{\prime}\right)\right) \varphi_{D_{r}, \theta^{\prime}}\left(a^{-1}\left(s+\theta-\theta^{\prime}\right)\right) \text {. }
$$

where $\theta^{\prime}=h^{-1}(\sin (\theta+\epsilon)+h(\theta))$.

Similarly, for all $\epsilon>0$ and s such that $\operatorname{sh}(\theta-\epsilon)+h(\theta) \epsilon h(\Theta)$, we have

$$
\varphi i_{\tau}(\theta-s), \theta(s)=\exp \left(a^{-1} u\left(s+\theta-\theta^{\prime \prime}\right)\right) \varphi_{D_{\tau}, \theta^{\prime \prime}}\left(a^{-1}\left(s+\theta-\theta^{\prime \prime}\right)\right),
$$

where $\theta^{\prime \prime}=h^{-1}(\sin (\theta-\epsilon)+h(\theta))$.

Proof. By (1.4) we have

$$
\dot{l}_{\tau}(\theta+\epsilon)=a^{-1}\left(u+D_{\tau}\right)-\dot{h}(\theta+\epsilon) Y(\tau)
$$

and

$$
\theta X(\tau)-f(\theta) Y(\tau)=a^{-1}\left(u+D_{\tau}\right) \theta-h(\theta) Y(\tau) .
$$

In view of (3.1) and (3.2) we have

$$
\begin{aligned}
\varphi i_{\tau}(\theta+\iota), \theta(s)= & E_{\theta}\left[\exp \left(s\left(a^{-1}\left(u+D_{\tau}\right)-\dot{h}(\theta+\epsilon) Y(\tau)\right)\right)\right] \\
= & E_{\mu_{\tau}}\left[g _ { \tau } \operatorname { e x p } \left(s \left(a^{-1}\left(u+D_{\tau}\right)\right.\right.\right. \\
& -\dot{h}(\theta+\epsilon) Y(\tau))) \exp (\theta X(\tau)-f(\theta) Y(\tau))] \\
= & E_{\mu_{\tau}}\left[g_{\tau} \exp \left((s+\theta) a^{-1}\left(u+D_{\tau}\right)-(\operatorname{sh}(\theta+\epsilon)+h(\theta)) Y(\tau)\right)\right] .
\end{aligned}
$$

Since $h$ is invertible on $\Theta$ and $\operatorname{sh}(\theta+\epsilon)+h(\theta) \epsilon h(\Theta)$, there exists a $\theta^{\prime} \in \Theta$ such that $h\left(\theta^{\prime}\right)=\operatorname{sih}(\theta+\epsilon)+h(\theta)$. Hence, we have

$$
\begin{aligned}
\text { R.H.S. of }(3.3)= & E_{\mu_{\tau}}\left[\exp \left((s+\theta) a^{-1}\left(u+D_{\tau}\right)-a^{-1}\left(u+D_{\tau}\right) \theta^{\prime}\right)\right. \\
& \left.\cdot g_{\tau} \exp \left(a^{-1}\left(u+D_{\tau}\right) \theta^{\prime}-h\left(\theta^{\prime}\right) Y(\tau)\right)\right] \\
= & E_{\theta^{\prime}}\left[\exp \left((s+\theta) a^{-1}\left(u+D_{\tau}\right)-a^{-1}\left(u+D_{\tau}\right) \theta^{\prime}\right)\right] \\
= & \exp \left(a^{-1} u\left(s+\theta-\theta^{\prime}\right)\right) E_{\theta^{\prime}}\left[\exp \left(a^{-1}\left(s+\theta-\theta^{\prime}\right) D_{\tau}\right)\right] \\
= & \exp \left(a^{-1} u\left(s+\theta-\theta^{\prime}\right)\right) \varphi_{D_{-}, \theta^{\prime}}\left(a^{-1}\left(s+\theta-\theta^{\prime}\right)\right) .
\end{aligned}
$$

Thus, the first asscrtion has been shown, and similarly, the second assertion is also shown. This completes the proof. 
Let $\quad \rho_{i_{r}(\theta+s), \theta}=\inf _{s} \varphi i_{\tau}(\theta+s), \theta(s)$ and $\rho_{i_{\tau}(\theta-s), \theta}=\inf _{s} \varphi i_{\tau}(\theta-s), \theta(s)$.

Lemma 3.2. Suppose that $P_{\theta}\left(\tau_{a, b}(u)<\infty\right)=1$ for all $\theta \in \Theta$ and condition (C) is fulfilled. Then for all sufficiently small $\epsilon>0$, we have

$$
\limsup _{u \rightarrow \infty} \frac{1}{u} \log \rho_{i_{\mathrm{r}}(\theta+\imath), \theta} \leq-K(\theta+\epsilon, \theta),
$$

and

$$
\limsup _{u \rightarrow \infty} \frac{1}{u} \log \rho_{i_{\tau}(\theta-s), \theta} \leq-K(\theta-\epsilon, \theta) .
$$

Proof. By Lemma 3.1 it follows that

$$
\log \varphi i_{\tau}(\theta+e), \theta(s)=a^{-1} u\left(s+\theta-\theta^{\prime}\right)+\log \varphi_{D_{\tau}, \theta^{\prime}}\left(a^{-1}\left(s+\theta-\theta^{\prime}\right)\right),
$$

where $\theta^{\prime}=h^{-1}(\operatorname{sh}(\theta+\epsilon)+h(\theta))$.

Let $\psi_{\theta, r}(s)=a^{-1} u\left(s+\theta-\theta^{\prime}\right)$. First we show that $\psi_{\theta, c}$ attains its minimum for a positive so. Since $h$ is invertible and differentiable on $\Theta$, we have

$$
\frac{\partial}{\partial s} \psi_{\theta, s}(s)=a^{-1} u\left(1-\frac{\dot{h}(\theta+\epsilon)}{\dot{h}\left(h^{-1}(\operatorname{sh}(\theta+\epsilon)+h(\theta))\right)}\right) \text {. }
$$

The equation $(\partial / \partial s) \psi_{\theta, \varepsilon}(s)=0$ has a unique solution $s_{0}=(h(\theta+\epsilon)-h(\theta)) / \dot{h}(\theta+\epsilon)$ $>0$. It is easy to see that $(\partial / \partial s) \psi_{\theta, s}(s)>0$ for $s>s_{0}$, and $(\partial / \partial s) \psi_{\theta, c}(s)<0$ for $s<s_{\text {o. }}$. Hence, we have

$$
\begin{aligned}
\inf _{s} \psi_{\theta, ،}(s) & =\psi_{\theta, s}\left(s_{0}\right) \\
& =a^{-1} u\left(s_{0}+\theta-\theta^{\prime}\right) \\
& =a^{-1} u\left(\frac{h(\theta+\epsilon)-h(\theta)}{\dot{h}(\theta+\epsilon)}+\theta-h^{-1}(h(\theta+\epsilon))\right) \\
& =\frac{a^{-1} u(h(\theta+\epsilon)-h(\theta)-\epsilon \dot{h}(\theta+\epsilon))}{\dot{h}(\theta+\epsilon)} \\
& =-u K(\theta+\epsilon, \theta) .
\end{aligned}
$$

From (3.4) and condition (C), we have

$$
\begin{aligned}
\frac{1}{u} \log \rho_{i_{\tau}(\theta+s), \theta} & =\inf _{s} \frac{1}{u} \log \varphi_{i_{\tau}(\theta+\varepsilon), \theta}(s) \\
& =\inf _{s}\left[\frac{1}{u} \psi_{\theta, \iota}(s)+\frac{1}{u} \log \varphi_{D_{\tau}, \theta^{\prime}}\left(a^{-1}\left(s+\theta-\theta^{\prime}\right)\right)\right] \\
& \leq \frac{1}{u} \psi_{\theta, \cdot(}\left(s_{0}\right)+\frac{1}{u} \log \varphi_{D_{\tau}, \theta^{\prime}}\left(a^{-1}\left(s_{0}+\theta-\theta^{\prime}\right)\right) \\
& \leq-K(\theta+\epsilon, \theta)+\frac{1}{u} \log E_{\theta^{\prime}}\left[e^{\left|a^{-1}\left(s_{0}+\theta-\theta^{\prime}\right)\right| M_{u}, \theta}\right] .
\end{aligned}
$$

For all sufficiently small $\epsilon>0, a^{-1}\left(s_{0}+\theta-\theta^{\prime}\right)$ is contained in the domain of the m.g.f. of $M_{u, \theta}$, and $\theta^{\prime}=h^{-1}\left(s_{0} \dot{h}(\theta+\epsilon)+h(\theta)\right) \in N_{\theta}$. Hence, by condition (C), the second term on the R.H.S. of (3.5) tends to 0 as $u \rightarrow \infty$. Therefore we have 


$$
\limsup _{u \rightarrow \infty} \frac{1}{u} \log \rho_{i_{\tau}(\theta+s), \theta} \leq-K(\theta+\epsilon, \theta) .
$$

Similarly, for all sufficiently small $\epsilon>0$, we have

$$
\limsup _{u \rightarrow \infty} \frac{1}{u} \log \rho_{i_{\tau}(\theta-\bullet), \theta} \leq-K(\theta-\epsilon, \theta) .
$$

This completes the proof.

Lemma 3.3. Suppose that $P_{\theta}\left(\tau_{a, b}(u)<\infty\right)=1$ for all $\theta \in \Theta$ and condition (C) is fulfilled. Let $T(\tau)$ be a consistent estimator of $\theta$ as $u \rightarrow \infty$. Then for any $\theta \in \Theta$ and $\epsilon>0$ such that $\theta \pm \epsilon \in \Theta$, we have

$$
\liminf _{u \rightarrow \infty} \frac{1}{u} \log P_{\theta, \tau}(|T(\tau)-\theta|>\epsilon) \geq-B(\theta, \epsilon) .
$$

Proof. For any $\delta>0$ and any $\epsilon^{\prime}>\epsilon$ such that $\theta \pm \epsilon^{\prime} \in \Theta$, we have

$$
\begin{aligned}
& P_{\theta, \tau}(|T(\tau)-\theta|>\epsilon)=\int_{[|T(\tau)-\theta|>\delta)} d P_{0, \tau}
\end{aligned}
$$

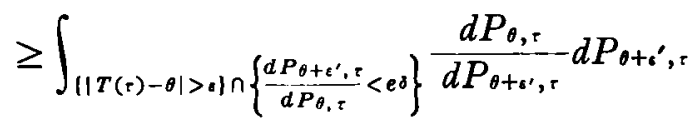

$$
\begin{aligned}
& \geq e^{-\delta} \int_{|| T(\tau)-\theta \mid>\varepsilon\} n\left\{\frac{d P_{\theta+\varepsilon^{\prime}, \tau}}{d P_{\theta, \tau}}<e^{\dot{o}}\right\}} d P_{\theta+\varepsilon^{\prime}, \tau} \\
& \geq e^{-\delta}\left(P_{\theta+\varepsilon^{\prime}, \tau}(|T(\tau)-\theta|>\epsilon)-P_{\theta+\varepsilon^{\prime}, \tau}\left(\frac{d P_{\theta+\varepsilon^{\prime}, \tau}}{d P_{\theta, \tau}}>e^{\delta}\right)\right) .
\end{aligned}
$$

Since $T(\tau)$ is a consistent estimator of $\theta$, we have

$$
\lim _{u \rightarrow \infty} P_{\theta+\theta^{\prime}, r}(|T(\tau)-\theta|>\epsilon)=1 .
$$

Put $\delta=u\left(K\left(\theta+\epsilon^{\prime}, \theta\right)+\delta_{1}\right)$, where $\delta_{1}$ is arbitrary positive number. Then it follows that

$$
\begin{aligned}
& P_{\theta+\varepsilon^{\prime}, \tau}\left(\frac{d P_{\theta+\epsilon^{\prime}, \tau}}{d P_{\theta, \tau}}>e^{\delta}\right) \\
&= P_{\theta+\epsilon^{\prime}, \tau}\left(\epsilon^{\prime} X(\tau)-\left(f\left(\theta+\epsilon^{\prime}\right)-f(\theta)\right) Y(\tau)>u\left(K\left(\theta+\epsilon^{\prime}, \theta\right)+\delta_{1}\right)\right) \\
&= P_{\theta+\epsilon^{\prime}, \tau}\left(\frac{a^{-1} \epsilon^{\prime}\left(D_{\tau}+u\right)}{u}-\left(a^{-1} b \epsilon^{\prime}+f\left(\theta+\epsilon^{\prime}\right)\right.\right. \\
&\left.\quad-f(\theta))-\frac{Y(\tau)}{u} \geq K\left(\theta+\epsilon^{\prime}, \theta\right)+\delta_{1}\right) \\
&= P_{\theta^{\prime}, \tau}\left(A_{\tau} \geq K\left(\theta+\epsilon^{\prime}, \theta\right)+\delta_{1}\right),
\end{aligned}
$$

where

$$
\theta^{\prime}=\theta+\epsilon^{\prime} \text { and } A_{\tau}=\frac{a^{-1} \epsilon^{\prime}\left(D_{t}+u\right)}{u}-\left(a^{-1} b \epsilon^{\prime}+f\left(\theta+\epsilon^{\prime}\right)-f(\theta)\right) \frac{Y(\tau)}{u} .
$$

We consider the m.g.f. of $Y(\tau) / u$ under $P_{\theta^{\prime}, \tau}$. In view of (3.2), we have 


$$
\begin{aligned}
\varphi_{Y(r) / u, \theta^{\prime}}(s)= & E_{\theta^{\prime}}\left[\exp \left(\frac{s Y(\tau)}{u}\right)\right] \\
= & E_{\mu_{\tau}}\left[g_{\mathrm{r}} \exp \left(a^{-1} \theta^{\prime}\left(u+D_{\tau}\right)-\left(h\left(\theta^{\prime}\right)-\frac{s}{u}\right) Y(\tau)\right)\right] \\
= & \exp \left(a^{-1} u\left(\theta^{\prime}-h^{-1}\left(h\left(\theta^{\prime}\right)-\frac{s}{u}\right)\right)\right) \\
& \cdot E_{\mu_{\tau}}\left[\exp \left(a^{-1}\left(\theta^{\prime}-h^{-1}\left(h\left(\theta^{\prime}\right)-\frac{s}{u}\right)\right) D_{\tau}\right)\right. \\
& \left.\cdot g_{\tau} \exp \left(a^{-1} h^{-1}\left(h\left(\theta^{\prime}\right)-\frac{s}{u}\right)\left(u+D_{r}\right)-\left(h\left(\theta^{\prime}\right)-\frac{s}{u}\right) Y(\tau)\right)\right] \\
= & \exp \left(a^{-1} u\left(\theta^{\prime}-h^{-1}\left(h\left(\theta^{\prime}\right)-\frac{s}{u}\right)\right)\right) \\
& \cdot E_{h^{-1}\left(n\left(\theta^{\prime}\right)-s / u\right)}\left[\exp \left(a^{-1}\left(\theta^{\prime}-h^{-1}\left(h\left(\theta^{\prime}\right)-\frac{s}{u}\right)\right) D_{\tau}\right)\right] \\
= & \exp \left(a^{-1} u\left(\theta^{\prime}-h^{-1}\left(h\left(\theta^{\prime}\right)-\frac{s}{u}\right)\right)\right) \\
& \cdot \varphi_{D_{r}, h^{-1}\left(h\left(\theta^{\prime}\right)-s / u\right)}\left(a^{-1}\left(\theta^{\prime}-h^{-1}\left(h\left(\theta^{\prime}\right)-\frac{s}{u}\right)\right)\right) .
\end{aligned}
$$

For $s$ in a neighbourhood of origin, from (1.5) we have

$$
\varphi_{D_{r}, h^{-1}\left(h\left(\theta^{\prime}\right)-s / u\right)}\left(a^{-1}\left(\theta^{\prime}-h^{-1}\left(h\left(\theta^{\prime}\right)-\frac{s}{u}\right)\right)\right) \rightarrow 1 \text { as } u \rightarrow \infty \text {. }
$$

Hence, we have

$$
\varphi_{Y(r) / u, \theta^{\prime}}(s) \rightarrow \exp \left(\frac{a^{-1} s}{\dot{h}\left(\theta^{\prime}\right)}\right) \quad \text { as } u \rightarrow \infty .
$$

By continuity theorem of m.g.f. it follows that

$$
-\frac{Y(\tau)}{u} \rightarrow \frac{a^{-1}}{\dot{h}\left(\theta^{\prime}\right)} \text { in probability as } u \rightarrow \infty \text { under } P_{\theta^{\prime}, r}
$$

From condition (C), $D_{r} / u \rightarrow 0$ in probability as $u \rightarrow \infty$ under $P_{\theta^{\prime}, r}$. Therefore, we have

$$
A_{\mathrm{r}} \rightarrow a^{-1} \epsilon^{\prime}-\left(a^{-1} b \epsilon^{\prime}+f\left(\theta^{\prime}\right)-f(\theta)\right) \frac{a^{-1}}{\dot{h}\left(\theta^{\prime}\right)}=K\left(\theta+\epsilon^{\prime}, \theta\right)
$$

in probability as $u \rightarrow \infty$ under $P_{\theta^{\prime}, r}$.

By (3.8), we have

$$
P_{\theta+\varepsilon^{\prime}, \tau}\left(\frac{d P_{\theta+\theta^{\prime}, \tau}}{d P_{\theta, \tau}}>e^{\delta}\right) \rightarrow 0 \text { as } u \rightarrow \infty .
$$

By (3.6) and (3.7), it follows that

$$
\liminf _{u \rightarrow \infty} \frac{1}{u} \log P_{\theta, \tau}(|T(\tau)-\theta|>\epsilon) \geq-K\left(\theta+\epsilon^{\prime}, \theta\right)-\delta_{1} .
$$

Since $\delta_{1}>0$ is arbitrary we have 


$$
\liminf _{u \rightarrow \infty} \frac{1}{u} \log P_{\theta, \mathrm{r}}(|T(\tau)-\theta|>\epsilon) \geq-K\left(\theta+\epsilon^{\prime}, \theta\right) .
$$

Moreover, since $\epsilon^{\prime}>\epsilon$ also is arbitrary we have

$$
\liminf _{u \rightarrow \infty} \frac{1}{u} \log P_{\theta, r}(|T(\tau)-\theta|>\epsilon) \geq-K(\theta+\epsilon, \theta) .
$$

By replacing $\theta+\epsilon^{\prime}$ with $\theta-\epsilon^{\prime}$ in the above discussions, we have

$$
\liminf _{u \rightarrow \infty} \frac{1}{u} \log P_{\theta, r}(|T(\tau)-\theta|>\epsilon) \geq-K(\theta-\epsilon, \theta) .
$$

From (3.9) and (3.10), the desired result is obtained. This completes the proof.

Proof of Theorem 2.1. By Corollary 1.1, the sequential maximum likelihood estimator $\hat{\theta}_{\mathrm{r}}$ is a consistent estimator of $\theta$. It follows that

$$
\begin{aligned}
P_{\theta, \tau}\left(\left|\hat{\theta}_{\tau}-\theta\right|>\epsilon\right) & =P_{\theta, \tau}\left(\hat{\theta}_{\tau}>\theta+\varepsilon\right)+P_{\theta, \tau}\left(\hat{\theta}_{\tau}<\theta-\epsilon\right) \\
& =P_{\theta, \tau}\left(i_{\tau}(\theta+\epsilon)>0\right)+P_{\theta, \tau}\left(i_{\tau}(\theta-\epsilon)<0\right) \\
& =I_{1}+I_{2},
\end{aligned}
$$

where

$$
I_{1}=P_{\theta, \tau}\left(\dot{l}_{r}(\theta+\epsilon)>0\right)
$$

and

$$
I_{2}=P_{\theta, \tau}\left(\dot{l}_{\tau}(\theta-\epsilon)<0\right) .
$$

By Markov inequality and Lemma 3.2, we have

$$
\begin{aligned}
\limsup _{u \rightarrow \infty} \frac{1}{u} \log I_{1} & \leq \limsup _{u \rightarrow \infty} \frac{1}{u}\left(\log \left(\inf _{s>0} E_{\theta}\left[\exp \left(s \dot{l}_{\tau}(\theta+\epsilon)\right)\right]\right)\right) \\
& =\limsup _{u \rightarrow \infty} \frac{1}{u} \log \rho_{i_{\tau}(\theta+\epsilon), \theta} \\
& \leq-K(\theta+\epsilon, \theta) .
\end{aligned}
$$

Similarly, we have

$$
\limsup _{u \rightarrow \infty} \frac{1}{u} \log I_{2} \leq-K(\theta-\epsilon, \theta) .
$$

From (3.12) and (3.13), for any $\delta>0$ it follows that for all sufficiently large $u>u(\delta), I_{1} \leq e^{(-B(\theta, \epsilon)+\delta) u}$ and $I_{2} \leq e^{(-B(\theta, \theta)+\delta) u}$.

Hence, by (3.11) we have

$$
\limsup _{u \rightarrow \infty} \frac{1}{u} \log P_{\theta, \mathrm{r}}\left(\left|\hat{\theta}_{\mathrm{r}}-\theta\right|>\epsilon\right) \leq-B(\theta, \epsilon)+\delta .
$$

Since $\delta>0$ is arbitrary, it follows that

$$
\limsup _{u \rightarrow \infty} \frac{1}{u} \log P_{\theta, r}\left(\left|\hat{\theta}_{\mathrm{r}}-\theta\right|>\epsilon\right) \leq-B(\theta, \epsilon) .
$$

Therefore, from Lemma 3.3 and (3.14), we have

$$
\lim _{u \rightarrow \infty} \frac{1}{u} \log P_{\theta, r}\left(\left|\hat{\theta}_{\mathfrak{r}}-\theta\right|>\epsilon\right)=-B(\theta, \epsilon) .
$$


This completes the proof.

\section{Examples}

Example 1. Let $X(t)$ be a Wiener process with drift $\theta$ and unit variance. The likelihood function is given by

$$
L_{\iota}(\theta)=\frac{1}{\sqrt{2 \pi t}} \exp \left(-\frac{X(t)^{2}}{2 t}\right) \exp \left(\theta X(t)-\frac{1}{2} \theta^{2} t\right) .
$$

where $\theta$ is a parameter varying in a subset $\Theta$ of $R^{1}$. Let $\Theta$ be a subset of $\{\theta$ : $a \theta+b>0\}$. The sequential maximum likelihood estimator $\hat{\theta}_{\tau}$ is given by

$$
\hat{\theta}_{\tau}=\frac{X(\tau)}{\tau} .
$$

In this case, since $X(t)$ and $Y(t)=t$ are continuous with probability one, it follows that $P_{\theta}\left(D_{\mathrm{t}}=0\right)=1$ for all $\theta \in \Theta$ and all $u>0$. Therefore, condition (C) is fulfilled.

Example 2. Let $X(t)$ be a Poisson process with intensity $\lambda>0$. The likelihood function is given by

$$
L_{t}(\theta)=\frac{t^{X(t)}}{X(t) !} \exp \left(\theta X(t)-e^{\theta} t\right), \quad t \in T=\{1,2, \cdots \cdots\}, \quad \theta \in \Theta,
$$

where $\theta=\log \lambda$, and $\Theta$ is a subset of the set $\left\{\theta: a e^{\theta}+b>0\right\}$. The sequential maximum likelihood estimator is given by $\hat{\theta}_{\mathrm{r}}=\log (X(\tau) / \tau)$. This process has jumps of size one. Hence, we have $0 \leq D_{\mathrm{r}} \leq|a|$ with probability one. It is easy to see that condition (C) is fulfilled.

Example 3. Consider the first order non-explosive Gaussian autoregressive process

$$
U_{t}=\theta U_{t-1}+\epsilon_{t}, \quad U_{0} \equiv 0, \quad|\theta|<1, \quad t \in T=\{1,2, \cdots \cdots\},
$$

where $\epsilon_{1}, \epsilon_{2}, \ldots$ are $N(0,1)$-distributed and independent. The likelihood function is given by

$$
L_{t}(\theta)=\left(\frac{1}{\sqrt{2 \pi}}\right)^{t} \exp \left(-\frac{1}{2} \sum_{i=1}^{t} U_{i}^{2}\right) \exp \left(\theta \sum_{i=1}^{t} U_{i} U_{i-1}-\frac{1}{2} \theta^{2} \sum_{i=1}^{t} U_{i-1}^{2}\right) .
$$

The process $a X(t)+b Y(t)=a \sum_{i=1}^{t} U_{i} U_{i-1}+b \sum_{i=1}^{t} U_{i-1}^{2}$ may jump far past $u$ before it stops. We suppose that $a \theta+b>0$. We consider the increments of the process $a X(t)+b Y(t)$,

$$
\begin{aligned}
a X(t)+b Y(t)-(a X(t-1)+b Y(t-1)) & =a U_{t} U_{t-1}+b U_{t-1}^{2} \\
& =(a \theta+b) U_{t-1}^{2}+a \epsilon_{t} U_{t-1} .
\end{aligned}
$$

Let $\tilde{U}_{t}=\left(\left(1-\theta^{2}\right) /\left(1-\theta^{2 t}\right)\right) U_{t}$. Then $\tilde{U}_{t}$ is $N(0,1)$-distributed variable for all $t$. Let $\left(\theta_{-}, \theta_{+}\right)$be any interval in $(-1,1)$. It follows that for all $\theta \in\left(\theta_{-}, \theta_{+}\right)$,

$$
\begin{aligned}
|a X(t)+b Y(t)-(a X(t-1)+b Y(t-1))| & \leq(a \theta+b) \frac{1-\theta^{2 t}}{1-\theta^{2}} \tilde{U}_{t-1}^{2}+\left|a \epsilon_{t}\right|\left(\frac{1-\theta^{2 t}}{1-\theta^{2}}\right)^{1 / 2}\left|\tilde{U}_{t-1}\right| \\
& \leq k_{1} \tilde{U}_{t-1}^{2}+k_{2}\left|\epsilon_{t}\right|\left|\tilde{U}_{t-1}\right|,
\end{aligned}
$$


where $k_{1}, k_{2}$ are constants. Since the distribution of $k_{1} \tilde{U}_{t-1}^{2}+k_{2}\left|\epsilon_{t}\right|\left|\tilde{U}_{\iota-1}\right|$ is independent of $t$ and $\theta \in\left(\theta_{-}, \theta_{+}\right)$, condition (C) is fulfilled.

\section{Acknowledgments}

The author is grateful to the referee for their helpful comments and detailed reading of the manuscript.

\section{REFERENCES}

[ 1 ] Basawa, I. V. and Prakasa Rao, B. L. S. (1980). Statistical Inference for Stochastic Processes, Academic Press.

[2] Różański. (1980). A modification of Sudakov's lemma and efficient sequential plans for the Ornstein-Uhlenbech process, Zastosowania Matematyki Applicationes Mathematicae, 17, 73-85.

[ 3 ] S $\phi$ rensen, M. (1986). On sequential maximum likelihood estimation for exponential families of stochastic processes, International Statistical Review, 54, 191-210.

[4] Stefanov, V. T. (1985). On efficient stopping times, Stochastic Process and their Applications, 19, 305-314.

[5] Wald, A. (1947). Sequential Analysis, Dover.

[6] Winkler, W. and Franz, J. (1979). Sequential estimation problems for the exponential class of processes with independent increments, Scandinavian Journal of Statistics, 6, 129-139.

[7] Winkler, W., Franz, J., and Küchler, I. (1982). Sequential statistical procedures for processes of the exponential class with independent increments, Mathematische Operationsforschung und Statistik, ser Statistics, 13, 105-119. 